\title{
Nonrigid-Earth Rotation Solution
}

\author{
P. Bretagnon, P. Rocher, J.-L. Simon
}

Institut de mécanique céleste, Observatoire de Paris, 77 avenue Denfert-Rochereau 75014 Paris

\begin{abstract}
The accuracy of the rigid-Earth solution SMART97 is $2 \mu$ as over the time interval $(1968,2023)$. To obtain a nonrigid-Earth solution, we use the transfer function of Mathews (1999). The perturbations of the third component of the angular velocity vector are taken into account.
\end{abstract}

\section{The rigid-Earth solution SMART97}

The differential equations of the rigid-Earth rotation are

$$
\begin{aligned}
\ddot{\omega} & =\frac{L}{A}+\frac{B-A}{B} \sin \tilde{\varphi}\left(\frac{M}{A} \cos \tilde{\varphi}-\frac{L}{A} \sin \tilde{\varphi}\right)-\dot{\psi} \dot{\varphi} \sin \omega \\
& -\frac{C-B}{A} \dot{\psi} \sin \omega(\dot{\varphi}+\dot{\psi} \cos \omega) \\
& +\frac{B-A}{A} \frac{C-A-B}{B} \times\left(\dot{\psi} \sin \omega \sin ^{2} \tilde{\varphi}+\frac{1}{2} \dot{\omega} \sin 2 \tilde{\varphi}\right)(\dot{\varphi}+\dot{\psi} \cos \omega), \\
\sin \omega \ddot{\psi} & =\frac{M}{B}+\frac{B-A}{A} \sin \tilde{\varphi}\left(\frac{M}{B} \sin \tilde{\varphi}+\frac{L}{B} \cos \tilde{\varphi}\right)+\frac{C+B-A}{B} \dot{\varphi} \dot{\omega} \\
& +\frac{C-A-B}{B} \dot{\psi} \dot{\omega} \cos \omega \\
& +\frac{B-A}{A} \frac{C-A-B}{B} \times\left(\dot{\omega} \sin ^{2} \tilde{\varphi}-\frac{1}{2} \dot{\psi} \sin \omega \sin 2 \tilde{\varphi}\right)(\dot{\varphi}+\dot{\psi} \cos \omega), \\
\ddot{\varphi} & =\frac{N}{C}-\ddot{\psi} \cos \omega+\dot{\psi} \dot{\omega} \sin \omega \\
& +\frac{B-A}{C}\left(\frac{1}{2} \dot{\omega}^{2} \sin 2 \tilde{\varphi}-\dot{\psi} \dot{\omega} \cos 2 \tilde{\varphi} \sin \omega-\frac{1}{4} \dot{\psi}^{2} \sin 2 \tilde{\varphi}(1-\cos 2 \omega)\right)
\end{aligned}
$$

The torque ( $L, M, N)$ has to be computed by using simultaneously the zonal and the tesseral harmonics because the first and the second derivatives of the diurnal and the semidiurnal terms are very important with respect to the longperiod terms. Table 1 gives the amplitudes of the semidiurnal term (coming from $C_{2,2}$ and $S_{2,2}$ ), of the 18.6-year term and of the 13.66-day term.

Integrated in this way, the rigid Earth solution SMART97 (Bretagnon et al., 1998) can reach a high accuracy. It has been compared with a numerical integration using DE403 (Standish et al., 1995) for the positions of the Moon, the Sun and the planets. The accuracy is $2 \mu$ as over 1968-2023. Figure 1 gives the differences for $\psi, \omega$ and for the Earth rotation angle $\varphi$. 

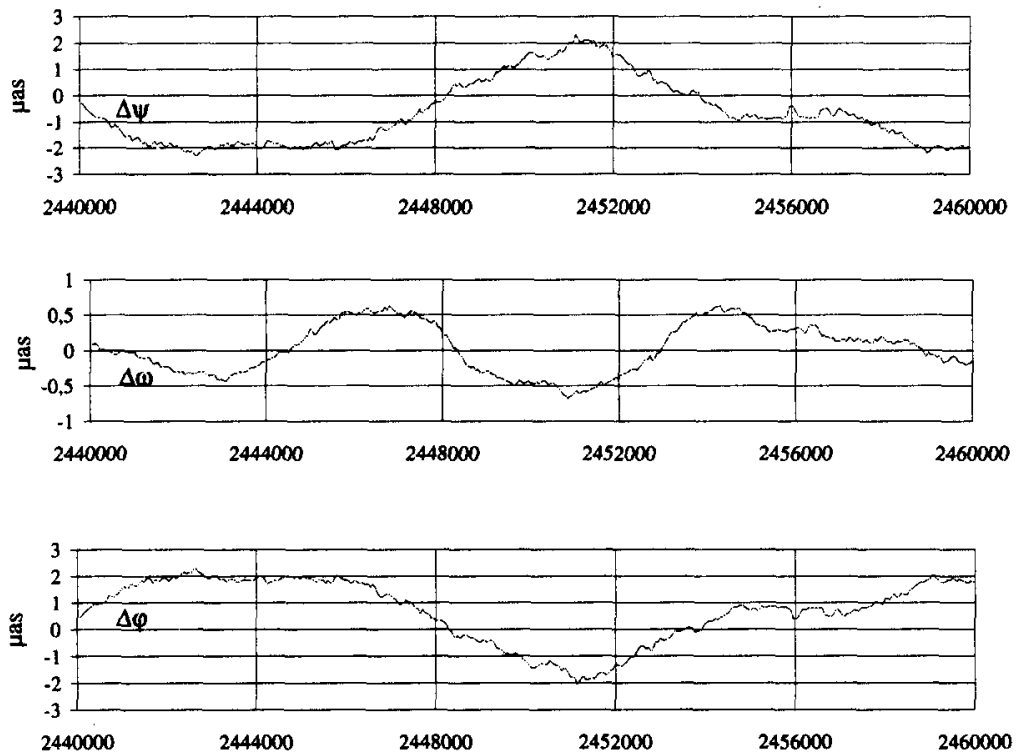

Figure 1. Theory SMART97 - numerical integration using DE403 over 1968-2023.

\section{The influence of the truncation level}

Table 2 gives the number of the periodic terms for $\sigma=0.01 \mu \mathrm{as}, \sigma=0.1 \mu \mathrm{as}$, and $\sigma=1 \mu \mathrm{as}$ and the number of Poisson terms greater than $\sigma$ over [J2000.0-100 yrs, $\mathrm{J} 2000.0+100 \mathrm{yrs}$ ], for the rigid-Earth solution SMART97. The table also gives the accuracy of the solution for different levels of truncation. Data are given for the variable $p(\Delta \psi$ in the IERS Conventions 1996) of nutation in longitude reckoned from the equinox of date. The number of terms of the Herring solution for a nonrigid Earth (McCarthy, 1996) is given by comparison.

Table 1. Amplitude of the 18.6-year, 18.66-day, 12-hour terms and of their first and second derivatives.

\begin{tabular}{lrrr} 
period & $\psi($ in $")$ & $\dot{\psi}($ in " $/ \mathrm{yr})$ & $\ddot{\psi}\left(\right.$ in $\left.^{\prime \prime} / \mathrm{yr}^{2}\right)$ \\
\hline 18.6 years & 17.280776 & 5.838 & 1.96 \\
13.66 days & 0.221507 & 37.212 & 6251.37 \\
12 hours & 0.000036 & 0.132 & 762.56 \\
\hline
\end{tabular}

The Poisson terms In SMART97, the P $\times t$ Poisson terms (Poisson terms of degree 1) represent $13.6 \%$ of the periodic terms with a truncation of $1 \mu$ as, $16.8 \%$ with a truncation of $0.1 \mu$ as and $20.8 \%$ with a truncation of $0.01 \mu$ as. That must be compared to the Herring solution in which the $\mathrm{P} \times \mathrm{t}$ Poisson terms represent $29.1 \%$ of the periodic terms with a truncation of $1 \mu$ as. 
The truncation In the construction of the SMART solution for a nonrigid Earth using the transfer function of Mathews, the amplitudes of some terms in resonance can be increased considerably. So, to obtain all the terms greater than 0.1 $\mu$ as in the nonrigid-Earth solution it is necessary to keep a level of truncation of $0.01 \mu$ as for the rigid-Earth solution. We give the number of terms of the SMART solution for a nonrigid Earth in Table 3.

Table 2. Number of terms of the nutation in longitude $p$ in SMART97 for different truncation levels $\sigma$.

\begin{tabular}{rcccccrr} 
Solution and $\sigma$ & $\mathrm{P}$ & $\mathrm{P} \times \mathrm{t}$ & $\mathrm{P} \times t^{2}$ & $\mathrm{P} \times t^{3}$ & $\mathrm{P} \times t^{4}$ & Total & Accuracy \\
\hline SMART (0.01 $\mu \mathrm{as})$ & 3910 & 815 & 183 & 14 & 2 & 4924 & $2.2 \mu \mathrm{as}$ \\
SMART (0.10 $\mu \mathrm{as})$ & 1586 & 266 & 61 & 4 & 0 & 1917 & $8 \mu \mathrm{as}$ \\
SMART (1.00 $\mu \mathrm{as})$ & 642 & 87 & 17 & 1 & 0 & 747 & $40 \mu \mathrm{as}$ \\
Herring (1.00 $\mu \mathrm{as})$ & 375 & 109 & 0 & 0 & 0 & 484 & \\
\hline
\end{tabular}

Table 3. Number of terms of the nutation in longitude of the nonrigid Earth solution at the $0.1 \mu$ as truncation level.

\begin{tabular}{cccccc} 
Periodic & $\mathrm{P} \times \mathrm{t}$ & $\mathrm{P} \times t^{2}$ & $\mathrm{P} \times t^{3}$ & $\mathrm{P} \times t^{4}$ & Total \\
\hline 1581 & 264 & 60 & 4 & 0 & 1909 \\
\hline
\end{tabular}

\section{Nonrigid-Earth solution}

\subsection{The transfer function of Mathews (1999)}

To compute the nonrigid-Earth solution, we use the transfer functions in a strict process. For instance, the transfer function of Mathews (1999) is

$$
T\left(\sigma ; e \mid e_{R}\right)=\frac{\left(e_{R}-\sigma\right)}{\left(e_{R}+1\right)} N_{0}\left(1+(1+\sigma) \sum_{\alpha} \frac{N_{\alpha}}{\sigma-\sigma_{\alpha}}\right),
$$

with $e_{R}=0.003284507,(1+\sigma)$ is the nutation frequency in space. We use the complex frequencies

$$
\begin{aligned}
& \sigma_{1}=(0.0025940,-0.0001438546), \sigma_{2}=(-1.00231861,0.00002578), \\
& \sigma_{3}=(-0.998957,0.000687), \quad \sigma_{4}=(0.000413499,0.000000280),
\end{aligned}
$$

and the complex coefficients

$$
\begin{aligned}
& N_{0}=\left(1.0000099,-0.37652854 \times 10^{-8}\right), \\
& N_{1}=(-0.79952969,0.043796154), \\
& N_{2}=\left(0.048964919,0.16332679 \times 10^{-2}\right), \\
& N_{3}=\left(0.29445472 \times 10^{-3},-0.82328898 \times 10^{-4}\right), \\
& N_{4}=\left(-0.15139223 \times 10^{-4},-0.11248592 \times 10^{-5}\right) .
\end{aligned}
$$




\subsection{Application to the Earth's angular velocity vector}

We apply the new transfer function not to the quantities $\sin \varepsilon_{0} \Delta \psi$ and $\Delta \varepsilon$ but to the Earth's angular velocity vector $(p, q, r)$ of the rigid case expressed as a function of the three Euler angles

$$
\begin{aligned}
& p_{R}=\dot{\psi}_{R} \sin \omega_{R} \sin \varphi_{R}+\dot{\omega}_{R} \cos \varphi_{R}, \\
& q_{R}=\dot{\psi}_{R} \sin \omega_{R} \cos \varphi_{R}-\dot{\omega}_{R} \sin \varphi_{R}, \\
& r_{R}=\dot{\psi}_{R} \cos \omega_{R}+\dot{\varphi}_{R} .
\end{aligned}
$$

For each prograde or retrograde argument, the angular velocity vector of the nonrigid Earth is obtained by multiplication by the transfer function

$$
\left(p_{N R}+i q_{N R}\right)=\left(p_{R}+i q_{R}\right) \times T\left(\sigma ; e \mid e_{R}\right) .
$$

From the quantities $\left(p_{N R}, q_{N R}, r_{N R}\right)$, we can compute the derivatives of $\psi, \omega$ and $\varphi$ in the nonrigid case by the inverse of (1)

$$
\begin{aligned}
\dot{\psi}_{N R} & =\left(p_{N R} \sin \varphi_{N R}+q_{N R} \cos \varphi_{N R}\right) / \sin \omega_{N R}, \\
\dot{\omega}_{N R} & =\left(p_{N R} \cos \varphi_{N R}-q_{N R} \sin \varphi_{N R}\right), \\
\dot{\varphi}_{N R} & =r_{N R}-\dot{\psi}_{N R} \cos \omega_{N R} .
\end{aligned}
$$

These equations are strict but the right-hand members depend on $\omega$ and $\varphi$ and the derivative of $\psi$, and we have to proceed by iterations to solve this system. The process converges without difficulties. The precision of the computation of $\dot{\psi}_{N R}$ is better than $3.5 \times 10^{-9}$ "/year which yields an accuracy of $0.01 \mu$ as for the 18.6-year term and $0.50 \mu$ as for the 883 year term. The classical method is to assume $r_{N R}=r_{R}$. The results are illustrated by the following table. We denote SM97M99 as the solution obtained by SMART97 + Mathews (1999). We can see the results from Mathews's function are very close to the series of Herring (McCarthy, 1996).

\begin{tabular}{lrrrrr} 
Solution & Argument & $p(\sin )$ & $p(\cos )$ & $\varepsilon(\sin )$ & $\varepsilon(\cos )$ \\
\hline SM97M99 & $\lambda_{3}+D-F$ & 17206664 & -3357 & -1488 & -9205156 \\
Herring & & 17206394 & -3702 & -1523 & -9205474 \\
SM97M99 & $2 \lambda_{3}$ & -1318625 & -662 & -486 & 573040 \\
Herring & & -1318526 & -670 & -471 & 573046 \\
SM97M99 & $2 \lambda_{3}+2 D$ & -227663 & 309 & 150 & 97854 \\
Herring & & -227720 & 269 & 136 & 97864 \\
SM97M99 & $\lambda_{3}$ & -36674 & -123068 & 16616 & 684 \\
Herring & & -36777 & -123010 & 16590 & 698 \\
\hline
\end{tabular}

The full method is to take into account the tidal variations in the Earth's rotation. We introduced the series $\left(\omega-\omega_{S}\right)$ and $\left(\omega-\omega_{D}\right)($ McCarthy, 1996) in order to obtain $r_{N R}$ from $r_{R}$,

$$
r_{N R}=r_{R}+\left(\omega-\omega_{S}\right)+\left(\omega-\omega_{D}\right) .
$$



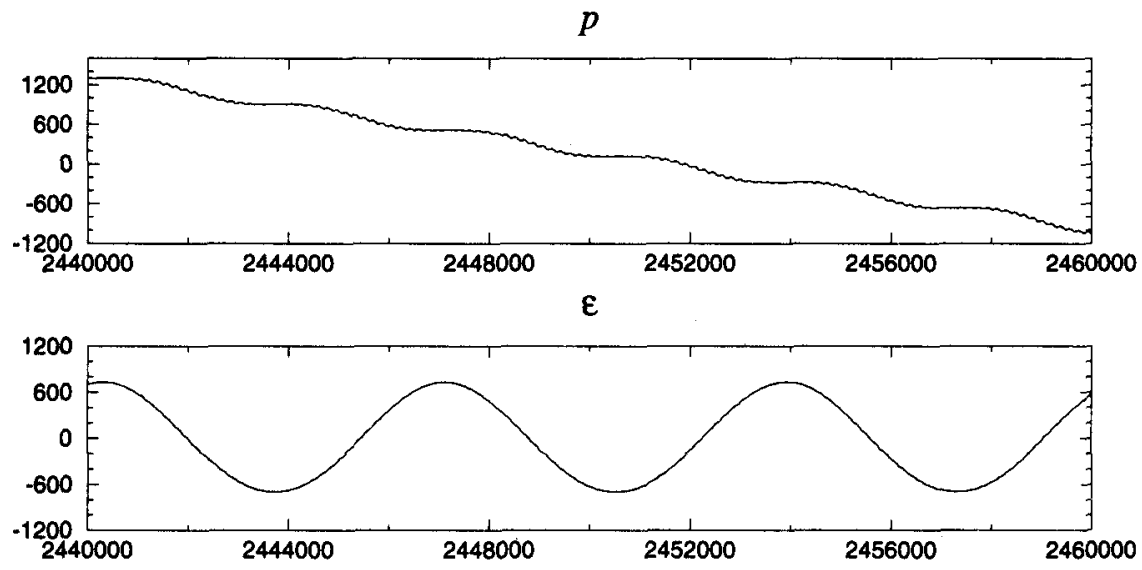

Figure 2. Influences of $\left(\omega-\omega_{S}\right)$ and $\left(\omega-\omega_{D}\right)$ on $p$ and $\varepsilon$ over 19682023. Units are $\mu$ as.

The modifications are very important not only for the third Euler angle $\varphi$ but also for the first two. For instance, we obtain

$$
\begin{aligned}
& \Delta p=42242 t+69 \sin \left(2 \lambda_{3}+2 D-2 F\right)-4 \sin \left(\lambda_{3}+D-F\right)+\cdots, \\
& \Delta \varepsilon=-15 t+712 \cos \left(\lambda_{3}+D-F\right)-19 \cos \left(2 \lambda_{3}+2 D-2 F\right)+\cdots,
\end{aligned}
$$

where the amplitudes are in $\mu$ as and the time in thousands of Julian years from J2000. Therefore, for $\varepsilon$, the 18.6-year term becomes

$$
\varepsilon=-1488 \sin \left(\lambda_{3}+D-F\right)-9204444 \cos \left(\lambda_{3}+D-F\right) .
$$

By comparison with the previous table we see the necessity to determine again the transfer function. The modifications of $p$ and $\varepsilon$ are plotted in Figure 2.

\section{Conclusion}

We have now to determine a new nonrigid-Earth rotation solution by using the new model of Mathews et al. (2000) taking into account the tidal variations in th Earth's rotation. 


\section{References}

Bretagnon, P., Francou, G., Rocher, P., Simon, J.-L., 1998, Astron. Astrophys., 329, 329.

McCarthy, D.D., 1996, IERS Standards (1996) IERS Technical Note 21, Observatoire de Paris.

Mathews, P.M., 1999, private communication.

Mathews, P.M., Herring, T.A., Buffet, B.A., 2000, New nonrigid Earth nutation series and Earth's interior, submitted to J. Geophys. Res.

Standish, E.M., Newhall, X X , Williams, J.G., Folkner, W.F., 1995, JPL IOM $314,10$. 